\title{
Human immunodeficiency virus impairs reverse cholesterol transport from macrophages Zahedi Mujawar ${ }^{1}$, Matthew P Morrow ${ }^{1}$, Dmitri Sviridov² and Michael Bukrinsky*1
}

Address: ${ }^{1}$ Department of Microbiology, Immunology and Tropical Medicine, George Washington University, Washington, District of Columbia, 20037, USA and ${ }^{2}$ Baker Heart Research Institute, Melbourne, Victoria 8008, Australia

* Corresponding author

from 2006 International Meeting of The Institute of Human Virology

Baltimore, USA. 17-2I November, 2006

Published: 21 December 2006

Retrovirology 2006, 3(SuppI I):S82 doi:10.1186/1742-4690-3-SI-S82

(c) 2006 Mujawar et al; licensee BioMed Central Ltd.

We demonstrate that HIV-1, via Nef, impairs ATP-binding cassette transporter A1 (ABCA1)-dependent cholesterol efflux from human macrophages. At least two mechanisms were involved: first, HIV infection and transfection with Nef induced post-transcriptional down-regulation of ABCA1; and second, Nef caused redistribution of ABCA1 to the plasma membrane and inhibited internalization of apolipoprotein A-I. Binding of Nef to ABCA1 was required for down-regulation and redistribution of ABCA1. Stimulation of cholesterol efflux from macrophages reduced infectivity of produced virions, and this effect correlated with a decreased amount of virion-associated cholesterol. Therefore, impairment of cholesterol efflux is essential to viral replication as it ensures proper cholesterol content in nascent HIV particles. This impairment may be a contributing factor to atherosclerosis in HIV patients. 